\title{
EXTRACELLULAR SUPEROXIDE DISMUTASE (EC-SOD) AS A PROTECTIVE FACTOR FOR RISK OF CHRONIC OBSTRUCTIVE PULMONARY DISEASE
}

R.P. Bowler*, J. Hokanson\#, M. Taylor", S. Levy, E.M. Canaham*, E. Regan*, C. Wheeler*, M. Nicks*,

E. Chan* and J.D. Crapo*

*Medicine, National Jewish Medical and Research Center, Denver, CO, USA

\#Preventive Medicine \& Biometrics, University of Colorado at Denver Health Sciences Center, Denver, CO, USA

'Medicine, University of Colorado at Denver Health Sciences Center, Denver, CO, USA

+Genetics, J. Craig Venter Institute, Rockville, MD, USA

WINNING ABSTRACT: Tobacco smoke contains a high concentration of oxidants and is the primary cause of chronic obstructive pulmonary disease (COPD). Extracellular superoxide dismutase (EC-SOD) is the major antioxidant enzyme in the extracellular space of the lung and is part of the lung defense against these oxidants. We hypothesized that EC-SOD is a risk factor for COPD. We found that EC-SOD plasma levels were significantly higher $(p<0.001)$ in 337 patients with COPD (147 \pm 7

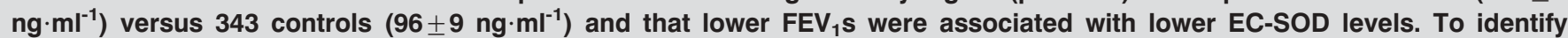
whether the EC-SOD gene was associated with COPD, we resequenced a subset of 188 subjects and identified 33 novel SNPs. Two of these SNPs (rs8192287 and rs8192288) were associated with a reduced odds of having COPD (OR 0.05 and 0.34 ; $\mathbf{P}<\mathbf{0 . 0 5}$ ). Haplotype analysis using a total of 5 EC-SOD SNPs (Table 1) further identified a protective haplotype (TTCGC) that was found in $11.4 \%$ of controls, but only $2.1 \%$ of subjects with COPD $(P<0.001)$. These data indicate that EC-SOD genotype may partially predict whether smokers are resistant to the effects smoking.

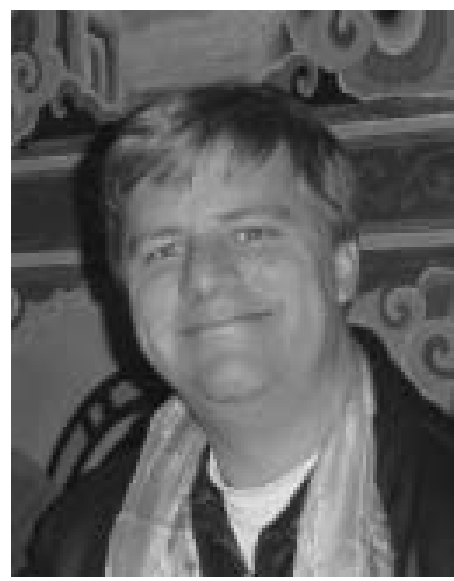

Russell P. Bowler

COPD Clinic, Division of Pulmonary Medicine, National Jewish Medical and Research Center, Denver, CO, USA

\section{MY JOB AND THE UNIT IN WHICH I WORK}

I work in the pulmonary division of the Dept of Medicine at the National Jewish Medical and Research Center in Denver, CO, USA. We are a speciality hospital and referral centre for pulmonary, allergic and immunological diseases. In 2005, we started a multidisciplinary programme in chronic obstructive pulmonary disease (COPD). The programme includes physicians, nurse practitioners, nurses, physical therapists, behavioural health specialists, nutritionists and pulmonary rehabilitation therapists, all of whom specialise in the care of COPD patients. We have several thousand visits per year for COPD. I am one of the founders, along with Barry Make, MD, a co-director of the clinic.

\section{MY WINNING POSTER AS PART OF MY RESEARCH}

We collect extensive phenotype data and blood samples on most of our COPD patients. Since most smokers do not develop COPD, there must be host factors (including genetic factors) that determine susceptibility to cigarette smoke. One of these factors is $\alpha_{1}$-antitrypsin (AT); however, only $1-2 \%$ of COPD patients have $\alpha_{1}$-AT deficiency. Extracelluar superoxide dismutase (EC-SOD) is one of the major antioxidant enzymes in the lung. Earlier this year, a polymorphism in EC-SOD was shown to decrease the odds of having COPD in residents of Copenhagen, Denmark [1]. We conducted additional work and identified two other polymorphisms that are protective of COPD in Denver, residents.

\section{MY RESEARCH AS PART OF MY WORKING GROUP/ RESEARCH TEAM}

This research fits well into the general theme of our laboratory, which is to discover biomarkers for COPD using genetic and

\begin{tabular}{lccc} 
TABLE 1 & $\begin{array}{l}\text { Major haplotypes and prevalences in COPD and } \\
\text { control subjects }\end{array}$ \\
EC-SOD haplotype & COPD & Controls & p-value \\
\hline G-G-C-A-C & 0.04 & 0.07 & 0.33 \\
G-G-C-G-C & 0.12 & 0.09 & 0.39 \\
G-G-C-G-T & 0.12 & 0.15 & 0.33 \\
G-G-T-A-C & 0.62 & 0.53 & 0.13 \\
G-G-T-G-C & 0.05 & 0.01 & 0.07 \\
G-G-T-G-T & 0.03 & 0.03 & 0.08 \\
T-T-C-G-C & 0.02 & 0.11 & $<0.001$ \\
\hline
\end{tabular}

Order of SNPs (left to right) is: rs8192287, rs8192288, rs699473, rs2536512, rs8192291. 
proteomic approaches. The work with EC-SOD is also particularly relevant because our laboratory group has extensive experience with oxidative stress and antioxidants in the lung. The group has used a wide variety of techniques to show that oxidative stress plays a major role in many pathological processes in the lung and that a primary role of the lungs antioxidant system is to defend these against oxidative insults. This work includes human, animal and cell cultures, and also includes the development of synthetic therapeutic antioxidants (e.g. metallophorphyrins).

THE IMPACT OF MY WORK ON CLINICAL OR RESEARCH PRACTICE

Genetic risk factors and protein biomarkers are desperately needed for COPD. Since so few smokers develop disease, identification of the early risk factors will permit more targeted interventions and reduce the size of clinical therapeutic studies. Furthermore, identification of these biomarkers will be likely to lead to improved understanding of the pathogenesis of cigarette smoking-related lung diseases. Ultimately, this knowledge can be used to develop new therapeutics, such as the class of antioxidants that we have been developing. This may lead to a reduction in morbidity and mortality in COPD patients.

\section{REFERENCES}

1 Juul K, Tybjaerg-Hansen A, Marklund S, Lange $P$, Nordestgaard BG. Genetically increased antioxidative protection and decreased chronic obstructive pulmonary disease. Am J Respir Crit Care Med 2006; 173: 858-864. 